KENTRON

REVUE PLURIDISCIPLINAIR

\title{
Kentron
}

Revue pluridisciplinaire du monde antique

$19 \mid 2003$

Le statut et l'image du corps dans la mythologie et la littérature grecques (suite et fin)

\section{Déni du corps et de la féminité}

\section{Gladys Johnston}

\section{(2) OpenEdition}

Journals

Édition électronique

URL : https://journals.openedition.org/kentron/1856

DOI : $10.4000 /$ kentron. 1856

ISSN : 2264-1459

Éditeur

Presses universitaires de Caen

\section{Édition imprimée}

Date de publication : 31 décembre 2003

Pagination : 137-142

ISBN : 2-84133-222-5

ISSN : 0765-0590

Référence électronique

Gladys Johnston, « Déni du corps et de la féminité », Kentron [En ligne], 19 | 2003, mis en ligne le 12 avril 2018, consulté le 13 octobre 2022. URL : http://journals.openedition.org/kentron/1856 ; DOI : https://doi.org/10.4000/kentron.1856

\section{(c) (i) (9)}

Creative Commons - Attribution - Pas d'Utilisation Commerciale - Pas de Modification 4.0 International - CC BY-NC-ND 4.0

https://creativecommons.org/licenses/by-nc-nd/4.0/ 


\section{DÉNI DU CORPS ET DE LA FÉMINITÉ}

Il y a 30 ans, en France, l'anorexie mentale des jeunes filles, et le terme même d'anorexie, étaient très peu connus du public et même des médecins. Avant le symposium sur l'anorexie mentale de 1965, elle était surtout une curiosité rare et constituait un objet de publication essentiellement des endocrinologues. Pourtant, cette affection est identifiée depuis 1689 .

À partir de 1965, la réflexion sur les anomalies des rapports de l'anorexique avec son corps et ses besoins ouvre de nouvelles perspectives. J'ai choisi de développer un aspect de la clinique de l'anorexique en référence au corps réel et imaginaire en dehors de sa dimension historique. Cette approche spécifique de la psychologie clinique concernant le corps apportera une lumière particulière propre à éclairer de nouveaux axes de réflexion.

Il semble pertinent de commencer par évoquer quelques points de compréhension de cette pathologie. La clinique de l'anorexie mentale est dominée par la triade symptomatique décrite par Lasègue en 1873 dite des $3 \mathrm{~A}$ : Anorexie, Amaigrissement, Aménorrhée.

- L'anorexie est une conduite volontaire de restriction alimentaire et de lutte contre la faim menée avec acharnement. C'est seulement après une longue période de restriction que la sensation de faim disparait, ce qui induit l'autorenforcement de la conduite. La restriction est qualitative et quantitative et aboutit à un régime dérisoire composé d'aliments soigneusement triés pour leur apport calorique minime, absorbés en petites quantités. D’abord masquée par des ruses ingénieuses, minimisée auprès de l'entourage qui s'inquiète, la conduite anorexique s'organise progressivement mais inéluctablement.

- L'amaigrissement est la conséquence directe de la conduite de restriction alimentaire. Une perte de poids de $15 \%$ par rapport au poids antérieur, en référence à la croissance normale, est considérée comme significative. Il n'est pas rare que la perte de poids atteigne 15 à $20 \mathrm{~kg}$ en quelques mois, donnant à l'adolescente un aspect cachectique et vieillot très impressionnant. Contrastant avec l'excessive maigreur, l'activité persiste: études, sorties, sport. On observe même une frénésie d'activités chez certaines patientes ne semblant ressentir ni la fatigue, ni le froid, ni le besoin de sommeil. Le sport, pratiqué intensément, fait également partie du processus d'amaigrissement en permettant de brûler des calories. 
- L’aménorrhée (disparition des règles) est constante. Elle peut précéder les autres symptômes ou s'installer dans les premiers mois d'évolution. Elle disparaît tardivement, plusieurs mois après le retour d'un poids normal.

Chez toutes les anorexiques, on retrouve la peur de grossir ainsi que la recherche d'une minceur idéale. Elles ont toutes, à un moment ou à un autre, restreint leur apport alimentaire pour perdre du poids, se jugeant trop grosses. Elles ne sont satisfaites ni par leur poids ni par leur morphologie. L'image qu'elles ont de leur corps n'est pas en rapport avec la réalité, elle est souvent déformée et dévalorisée. Le corps est rejeté, maltraité, ses dimensions sont surestimées, tout particulièrement les parties corporelles considérées comme spécifiquement féminines. La représentation du corps peut être lacunaire, ses limites peuvent être floues, l'intérieur ressenti comme vide. Ce corps ne procure pas de plaisir et la patiente, en tentant de le corriger, espère trouver une meilleure estime de soi. Elle corrige l'apparence extérieure par l'exercice physique intense, mais aussi l'intérieur en voulant le vider, l'épurer, le faire dégonfler, en se faisant vomir ou en avalant des laxatifs à haute dose. L'anorexique dénie les besoins fondamentaux de son corps et joue avec sa santé et sa vie de la façon la plus préoccupante qui soit.

Au point de vue psychologique les éléments les plus pertinents à retenir sont de plusieurs ordres :

- Présence de troubles de l'image du corps et de ses besoins, dominés par une peur intense, infondée et permanente de grossir, une méconnaissance et un refus de reconnaître la maladie ainsi que les besoins et les limites du corps. Il y a un rejet massif du corps et des transformations qu'impose la puberté avec un refus de la sexualité génitale. Du fait de sa métamorphose rapide et apparente, la fillette devient une adolescente qui suscite les regards masculins sur son corps qu'elle perçoit comme une intrusion persécutoire plutôt que comme une gratification narcissique. L'anorexique refuse donc ce corps féminin et tente de lui conserver cet aspect enfantin protecteur. Ainsi, à travers le rejet du corps et de ses besoins s'expriment l'incapacité d'intégrer les transformations physiques et affectives de la période pubertaire et le refus de la sexualité génitale.

- Présence de troubles majeurs du registre affectif et de l'identité. Derrière une pseudo-adaptation sociale souvent assez longtemps préservée, les sentiments de vide, d'impuissance et les doutes innombrables en sont le reflet et conduisent dans les formes évoluées à un repli progressif, souvent rationalisé par les exigences du travail scolaire (examens, contrôles...). L'ascétisme et l'intellectualisation, banals à l'adolescence, sont ici poussés à l'extrême. Le corps souhaité, fantasmé est un corps mince, beau, séducteur et sans défaut. Le corps réel, toujours vécu comme insatisfaisant, n’est pas appréhendé dans son apparence objective. L'anorexique dénie sa maigreur et même lorsqu'elle atteint un état cachectique, elle se trouve encore trop grosse, trop lourde. L'image du corps est donc complètement déniée au profit d'une néo-réalité pouvant engager le pronostic vital. 
- Présence d'une situation de conflit avec l'entourage familial au sein duquel s'organise la conduite. Avec des mouvements très ambivalents, à la fois de recherche d'affection et de combat pour une identité autonome, débouchant bientôt sur un grisant pouvoir de manipulation. Pouvoir refuser de se nourrir, c'est se sentir autonome, c'est s'assurer que l'on peut fonctionner sans apport extérieur conformément à une vision idéale de soi où le corps, nié dans sa réalité honteuse, fantasmé en corps désincarné, immortel, est constamment maîtrisé. De même qu'à travers la conduite anorexique, l'entourage est constamment contrôlé, défié et en particulier la mère. En luttant contre la faim et en faisant échec à ses pulsions, l'anorexique éprouve un sentiment de puissance et de domination sur elle-même et sur ses proches dont elle répudie les intérêts qu'elle juge matériels et grossiers. La satisfaction intense qu'elle en retire est de l'ordre de la jouissance perverse et cet auto-érotisme constitue un obstacle majeur au traitement. Enfin, chez l'anorexique, les images parentales demeurent imprécises et indifférenciées avec la prévalence d'une image maternelle omnipotente et asexuée.

- Présence d'une attitude distordue vis-à-vis de la nourriture et de l'alimentation. Cette attitude déborde largement la restriction volontaire et sélective de nourriture pour intégrer des préoccupations paradoxales incessantes la concernant (vol, stockage de nourriture, préparations de repas pour les autres, collection de fiches de recettes...)

Après l'exposé de ces éléments majeurs de compréhension de la conduite anorexique, il serait souhaitable de revenir plus longuement sur la notion d'ascétisme évoquée antérieurement et plus particulièrement sur celle de jeûne et de purification.

Le jeûne, par définition, c'est l'abstinence d'aliments et, par extension, la privation d'une chose dont on a besoin et toute privation qu'elle soit liée à des prescriptions religieuses ou à des comportements pathologiques. Cependant, l'anorexie étant de mieux en mieux identifiée comme restriction alimentaire pathologique, le jeûne revêt un sens de plus en plus limité à la normalité, en référence à l'hygiénisme d'une part et à la religion d'autre part: c'est un moyen pour la santé ou le salut (pratiqué dans la plupart des religions).

La comparaison avec l'anorexie mentale est d'autant plus opportune que l'on admet pour celle-ci un facteur culturel ou même que certains auteurs la définissent comme syndrome lié à la culture du fait de sa parenté avec les soucis féminins de régime, de poids si prégnants à l'heure actuelle. On peut donc comparer jeûne et anorexie mentale dans le but de tenter d'éclairer le normal par le pathologique et réciproquement de la même façon que Freud a rapproché le deuil et la mélancolie, le rêve et le délire.

Après avoir envisagé divers types de jeûne, la comparaison avec l'anorexie va nous conduire à mettre en évidence les thèmes du contrôle de soi et de la purification qui leur sont communs. 
L'anorexie, cliniquement, c'est le jeûne impossible:

- L’un, le jeûne, est social. Il se réfere à une culture, des croyances, un idéal partagé, se donnant comme un choix volontaire, un moyen non limité à ses effets sur le corps. C'est un acte de portée symbolique en rapport à des valeurs supérieures sinon transcendantes. Mais il comporte aussi la recherche du contrôle et du pouvoir sur soi, sur autrui, voire sur Dieu.

- L'autre, l'anorexie mentale, est solitaire, stéréotypée dans ses modalités, s'avouant tôt ou tard comme une contrainte subie en rapport avec des angoisses, un désarroi sans autre issue, la hantise incessante de grossir, de perdre le contrôle. La recherche de soi et du contrôle sur soi passe par le désir d'affranchissement visà-vis du corps. L'idéal n'est donc qu'en partie semblable.

La différence est claire:

- Le jeûne s'inscrit dans le rapport aux normes, aux usages, aux prescriptions, en fonction des appartenances, des allégeances, des solidarités, des traditions plus ou moins valorisées ou désaffectivées mais maintenues par habitude; bien des restrictions alimentaires apparaissent ainsi comme des rituels religieux laïcisés, mais le jeûne religieux ne trouve sens qu'en référence à Dieu.

- La restriction anorexique est, explicitement ou implicitement, rupture, opposition et révolte. Sont typiques l'intolérance aux restrictions alimentaires des autres, surtout des proches, le désir d'être la seule, la plus mince, la première : ce n'est pas un rite mais, au moins au début, une lutte et surtout un processus autocentré qui contraint le sujet. La privation, d'abord pénible, devient plus ou moins rapidement aisée : elle est perçue comme libératrice, comme condition de la réalisation d'un état de soi, d'une performance physique, intellectuelle. Elle permet de se rapprocher d'un idéal qui est à la fois esthésique (se sentir bien), esthétique (être belle, distinguée) et éthique (capable de volonté, de contrôle et de force). Il ne s'agit pas de sanctifier le corps pour sacraliser l'être mais de faire disparaître le corps pour être.

D’une manière générale, le jeûne crée la rupture avec la nature pour atteindre la culture, l'humanité, la société et la religion qui relie et ouvre à la dimension de la transcendance. Les fonctions répressives et sublimatoires de la morale et de la religion font du jeûne et de l'abstinence des moyens pour la domination des instincts, la maîtrise de soi renversant en son contraire le péché de gourmandise. Mais le but est aussi le renoncement à soi, le sacrifice, l'abnégation dans la soumission à l'idéal, à Dieu.

Il semble maintenant pertinent de distinguer les principales formes de jeûne afin d'en clarifier les liens avec l'anorexie.

- Le jeûne comme discipline: Socrate et Platon étaient des jeûneurs réguliers, jusqu'à dix jours de suite, pour conserver un bon état physique et mental. Pythagore exigeait de ses élèves 40 jours de jeûne avant de les admettre dans sa classe : la discipline alimentaire devait donner accès à la discipline intellectuelle. 
- Le jeûne comme pouvoir : le pouvoir réel et magique du jeûne a été fortement illustré par Gandhi : par son jeûne, il voulait purifier l'Inde, faire triompher la nonviolence, mais aussi mettre en échec le pouvoir colonial anglais. Jeûne magique et jeûne de protestation sont associés. Dans la Bible, le roi David jeûne douze jours parce que son fils est malade et l'enfant guérit. La grève de la faim est le dernier pouvoir inaliénable, le seul dont dispose le sujet.

- Le jeûne comme art de bien vivre : l'hygiénisme. Définie par l'OMS comme "état complet de bien-être physique et mental», la santé remplace le salut d'où la mystique laïque du jeûne. Dans cette religion laïque, la réduction des besoins, le vide digestif et le vide de l'esprit permettent le ressourcement de l'âme. La critique de la civilisation de l'abondance, de la surconsommation, de l'excès alimentaire aboutit à la conclusion insistante selon laquelle on mange trop dans les pays riches et qu'il est nécessaire de revenir aux valeurs premières, au jeûne, afin de retrouver la force vitale propre à maintenir en bonne santé.

- Le jeûne dans la médecine ayur-védique hindoue : elle ne vise rien de moins que la régénération, le rajeunissement sinon l'immortalité par l'élimination, l'épuration, notamment celle des toxines censées s'accumuler dans le corps du fait de l'ingestion de viande. D'où le recours à la purgation, à la purification et à la vomification. La purge par en haut et par en bas, de quoi séduire plus d'une anorexique.

- Le jeûne religieux: il a une double dimension, mystique et rituelle par les normes, les interdits et les prescriptions. Mais on retrouve toujours les thèmes de sacrifice, purification, pureté physique et morale ou encore le vide comme condition de remplissement mystique. Pour le zen, le taoïsme et la philosophie bouddhiste, le jeûne vise à augmenter la présence de soi, la conscience comme fondement du sentiment de soi dans des limites reconnues et dépassées. Dans le christianisme, le jeûne a pour fonction essentielle la purification qui rend la créature pécheresse digne de l'intimité avec son créateur. Elle rachète le péché originel d'Adam et Ève. Dans tous les cas, le jeûne religieux permet le renoncement au plaisir et au désir et le contrôle de soi.

Nous pouvons maintenant conclure que le jeûne vise à dominer les passions mauvaises et qu'il consiste en un sacrifice de soi. Les éléments communs avec l'anorexie sont légion et reposent essentiellement sur le thème du contrôle de soi et de la purification.

Dans l'anorexie mentale, outre le contrôle de soi et des autres par la maîtrise du corps et la restriction alimentaire, la purification est aussi recherchée par l'usage de laxatifs, de lavages compulsifs et de potomanie. La graisse est appréhendée comme souillure répugnante, l'obésité comme laideur et perte totale de valeur à la mesure de la démesure de l'idéal corporel angélique, léger, évanescent.

La restriction alimentaire du jeûne, par-delà sa signification d'appartenance religieuse, est conforme à des normes, à des préceptes, avec ou sans croyances. Dans 
l'anorexie, le caractère de contrainte, d'obligation, peut être considéré comme ayant une fonction fondamentale de soulagement de la culpabilité inconsciente. L'acte alimentaire vaut comme une consommation illustrant d'autres réalisations de désir : la sexualité et la haine. Manger, c'est inévitablement ingérer ce que l'on aime et simultanément le détruire: la punition, c'est l'empoisonnement, les toxines, le grossissement, mais surtout c'est la perte de contrôle et le débordement pulsionnel. Le jeûne apparaît donc comme traitement social de la culpabilité liée aux fantasmes inconscients qui peut, dans certains cas, déterminer l'anorexie.

Gladys Johnston

Université de Caen Basse-Normandie

\section{Références bibliographiques}

Aulagnier P. (1979), Les Destins du plaisir : aliénation, amour, passion, Paris, PUF.

Bateson G. (1980), Vers une écologie de l'esprit, Paris, Seuil.

BeLl R.M. [1985], L'Anorexie sainte, Paris, PUF, 1994.

Brusset B. (1990), «La jouissance de l'anorexique », Revue française de psychanalyse, LIV, 2.

Brusset B. (1994), «Le jeûne et la purification », Nervure, VII, 6, p. 9-15.

Brusset B. (1998), Psychopathologie de l'anorexie mentale, Paris, Dunod (Psychismes).

Снaвert C. (1998), «Un corps sacrifié», Les Cahiers du Centre de psychanalyse et de psychothérapie, 16-17, p. 35-49.

Freud S. [1924], «Le problème économique du masochisme», in Résultats, idées, problèmes, t. II, Paris, PUF, 1985.

LASÈgue C. (1873), «De l'anorexie hystérique », Archives générales de médecine, 1, p. 385-403. Samuel-Lajeunesse B., Foulon C. (1994), Les Conduites alimentaires, Paris, Masson. 